Anaesthesist 2006 · 55:541-546 DOI 10.1007/s00101-006-0974-y

Online publiziert: 24. Januar 2006

๑) Springer Medizin Verlag 2006
O. Juri · D. Frochaux ${ }^{2}$ - G. P. Rajan ${ }^{3}$ - P. Biro ${ }^{1}$

${ }^{1}$ Institut für Anästhesiologie, Universitätsspital, Zürich

${ }^{2}$ Institut für Anästhesiologie, Kantonsspital, Baden

${ }^{3}$ Dept. of Otolaryngology, Head \& Neck Surgery, Lions Ear and

Hearing Institute, University of Western Australia, Fremantle

\title{
Entflammungs- und Brandverhalten von biologischem Gewebe bei In-vitro-Bestrahlung mit dem $\mathrm{CO}_{2}$-Laser
}

Bei Eingriffen an den Atemwegen wird häufig ein $\mathrm{CO}_{2}$-LASER (Akronym für "light amplification by stimulated emission of radiation") eingesetzt. Vorteile für den Operateur liegen dabei in der exakteren Schnittführung, der geringeren Blutungstendenz in situ und der verminderten mechanischen Belastung, Ödembildung und Vernarbung des betroffenen Gewebes $[2,5,11,15]$. Ein gewichtiger Nachteil ist jedoch die Gefahr einer Entflammung oder eines Brandes in den Atemwegen und der Lunge des $\mathrm{Pa}$ tienten $[3,12]$. Der Laserstrahl kann entweder von außen eingebrachtes Material, wie Tuben, Katheter, Tupfer usw., in Brand setzen oder brennbares körpereigenes Material, wie eingetrocknetes Blut und Gewebepartikel. Laut älterer Literatur ist mit einer Häufigkeit von $0,4-1,5 \%$ von Brandzwischenfällen mit Laseranwendung bei Atemwegseingriffen zu rechnen [7]. Um einen Brand auszulösen, braucht es grundsätzlich drei Komponenten:

1. Brennstoff,

2. Oxidationsmittel und

3. Aktivierungsenergie [6].

Während das Fremdmaterial vom Operateur und Anästhesisten unter Berücksichtigung der Brandgefahr ausgewählt werden kann, gilt dies nicht für die Zusammensetzung des involvierten körpereige- nen Gewebes. Vereinzelte anekdotische (nichtpublizierte) Mitteilungen über Entflammungen unter Hochfrequenz-Jetventilation bei Verwendung nichtbrennbarer Katheter aus Teflon haben den Verdacht erhärtet, dass auch körpereigenes Gewebe unter bestimmten Umständen entzündet werden kann. Unter Jetventilation werden Gewebepartikel in einer sauerstoffhaltigen Atmosphäre aufgewirbelt, sodass sich ein entflammbares, möglicherweise sogar explosives Aerosol („Lasersmog“) bilden kann. Der Strahl des $\mathrm{CO}_{2}$-Lasers verdampft das Wasser im bestrahlten Gewebe, das dadurch komplett austrocknet. Der austretende Dampf versprüht feine fett- und eiweißhaltige Partikel in die unmittelbare Umgebung, die weitgehend aus dem nunmehr dehydrierten Zellmaterial des getroffenen Gewebes bestehen. Darunter sind kohlenstoffhaltige organische Verbindungen, die in Gegenwart von Sauerstoff und genügender Aktivierungsenergie brennbar sind $[9,10,17]$.

Um die Gefahr eines Atemwegsbrandes und das Entflammungsverhalten eines brennstoffreichen Lasersmogs zu untersuchen, haben wir eine In-vitro-Untersuchungsreihe durchgeführt, bei der wir organisches Gewebe unter Sauerstoffzufuhr über einen Jetventilationskatheter mit einem Laserstrahl bestrahlt haben. Dabei wurden Bedingungen simu- liert, wie sie auch in der klinischen Anwendung dieser Operations- und Beatmungstechnik vorkommen können bzw. darüber hinaus gehen.

\section{Methodik}

In Anlehnung an die Europäische Norm ISO-1199o wurde eine Stahlkammer konstruiert, um die Gaszusammensetzung im Hypopharynx und deren dynamische Veränderungen unter Jetbeatmung möglichst realitätsnah simulieren zu können. Die Kammer besteht aus einem Stahlzylinder mit einem Innendurchmesser von $10 \mathrm{~cm}$ und einer Länge von $15 \mathrm{~cm}$. Die Wandstärke beträgt überall $3 \mathrm{~mm}$. Das distale Ende des Zylinders ist mit einer Stahlplatte abgeschlossen, deren überstehende Ecken als Stützen gegen seitliches Wegrollen dienen. In die Oberseite der proximalen Kante ist eine Aussparung geschnitten, um den Laserstrahl in einem steilen Winkel in die Kammer einbringen zu können. Das Volumen des Zylinderinhalts $\left(\pi \times r^{2} \times h\right)$ ergibt $1177 \mathrm{~cm}^{3}$. Dieses ist zwar erheblich mehr als ein mit dem Kleinsasserspatel instrumentierter Hypopharynx (ca. $500-600 \mathrm{~cm}^{3}$ ), reduziert sich aber auf ein natürliches Maß, nach-

Diese Arbeit stellt die Ergebnisse der Dissertation von O. Juri dar. 


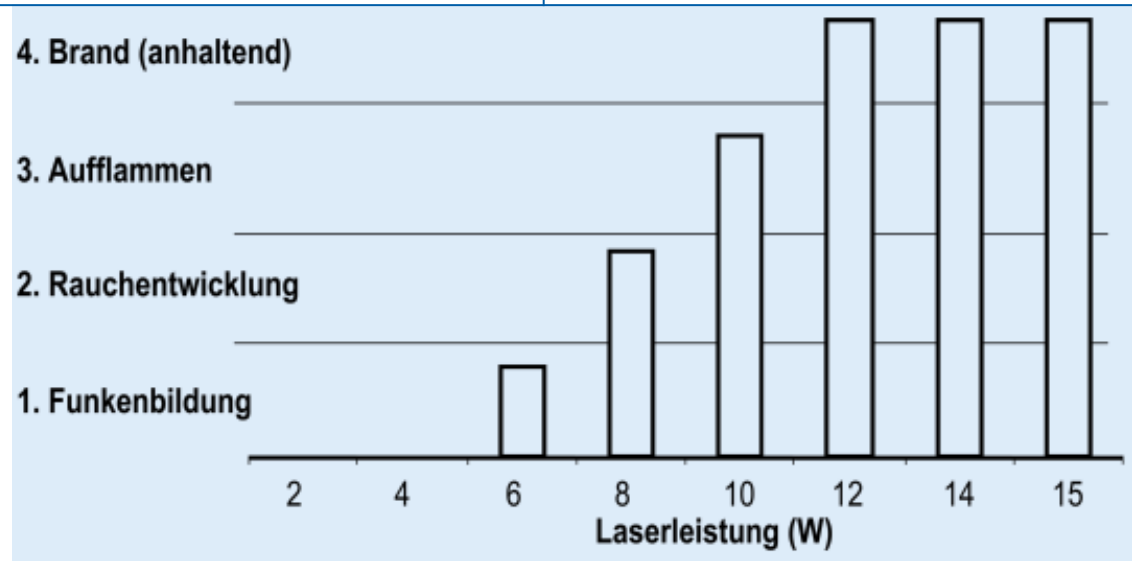

Abb. 1 A Art der Entzündungs- bzw. Brandphänomene bei Muskelgewebe in Abhängigkeit von der Laserleistung. Rahmenbedingungen: $75^{\circ}$-Auftreffwinkel auf Gewebe, Gasfluss durch den Jetkatheter $20 \mathrm{l} / \mathrm{min}$, Sauerstoffkonzentration $100 \%$, Bestrahlungsdauer $30 \mathrm{~s}$

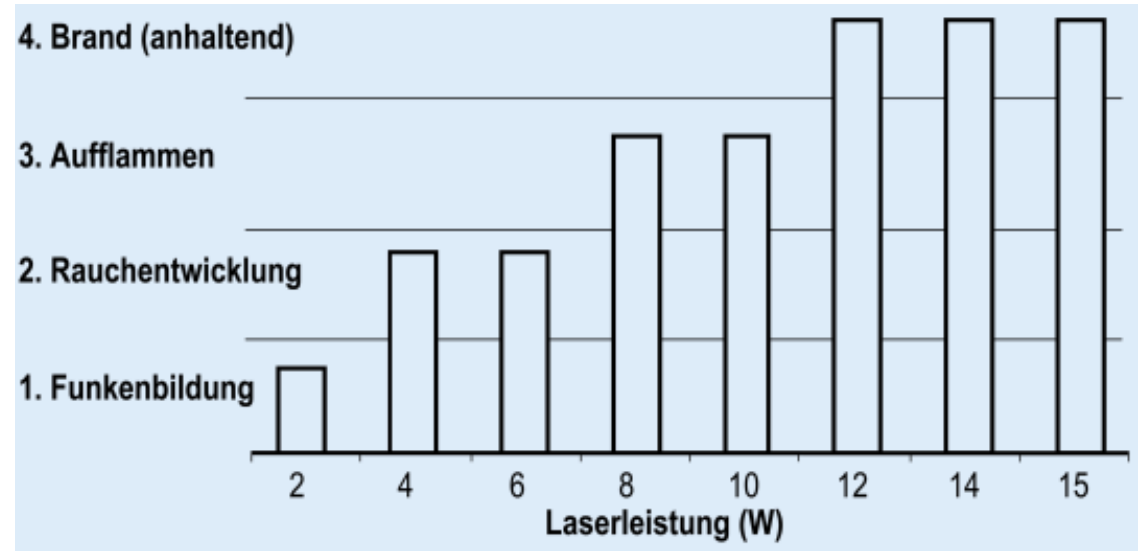

Abb. $2 \Delta$ Art der Entzündungs- bzw. Brandphänomene bei Fettgewebe in Abhängigkeit von der Laserleistung. Rahmenbedingungen: $75^{\circ}$-Auftreffwinkel auf Gewebe, Gasfluss durch den Jetkatheter $20 \mathrm{l} / \mathrm{min}$, Sauerstoffkonzentration $100 \%$, Bestrahlungsdauer $30 \mathrm{~s}$

dem genügend große Gewebeproben in die Stahlkammer eingeführt worden sind. Bei Letzteren handelt es sich um $2,5 \mathrm{~cm}$ dicke Scheiben rohen Schweinefleisches, die explizit für diese Versuchsreihe ausgewählt wurden, weil sie Muskel-, Fett-, Knochen- und Knorpelbestandteile beinhalten und damit bezüglich der Beschaffenheit eine große Ähnlichkeit mit dem Gewebe im Operationsgebiet aufweisen. Ein LaserJet -Katheter wurde an die Jetleitung eines Monsoon ${ }^{\circ}$-Jetventilators (Acutronic Medical Systems, Baar, Schweiz) angeschlossen. Das distale Ende des Jetkatheters wurde $10 \mathrm{~cm}$ tief in die Stahlkammer in einer Entfernung von $10 \mathrm{~mm}$ über dem Gewebe gelegt. Der Laserstrahl wurde mit einem „ESC/Sharplan $40 \mathrm{OC} \mathrm{CO}_{2}-$ Laser" (Lumenis GmbH, D-64807 Dieburg) erzeugt, der im klinischen Einsatz für mikrolaryngoskopische Eingriffe steht. Der Laserstrahl wurde im „Super-
puls-Modus“(Impulsrate $250 \mathrm{~Hz}$ ) abgegeben und mit einem Auftreffwinkel von $75^{\circ}$ auf das Gewebe gerichtet.

$\mathrm{Zu}$ Beginn wurde festgestellt, welche Entflammungs- und Brandphänomene $\mathrm{zu}$ erwarten waren, wenn ein genügend intensiver Laserstrahl mit einer Leistung von $10 \mathrm{~W}$ ohne Zeitlimite auf verschiedene Stellen des biologischen Gewebes appliziert wird. Um den Einfluss von Applikationsdauer und Laserintensität zu bestimmen, wurden anschließend 2 Versuchsreihen mit steigender Laserleistung von $2-15 \mathrm{~W}$ (in 2-W-Schritten bis $14 \mathrm{~W}$ ) durchgeführt. Dies geschah jeweils einmal mit Bestrahlung von Muskelgewebe und einmal mit Fettgewebe. Durch den Jetkatheter wurde ein Sauerstofffluss von 20 1/ $\mathrm{min}$ in einem Abstand von $10 \mathrm{~mm}$ vor die Auftreffzone des Laserstrahls geleitet.

In einer weiteren Versuchsreihe wurde die Zeitdauer visuell mit Stoppuhr er- mittelt, die bei Flutung der Versuchskammer mit 201 Sauerstoff/min erforderlich ist, bis eine Entflammung ausgelöst werden kann. Dabei wurde die Laserleistung von 2-15 W (in 2-W-Schritten bis $14 \mathrm{~W}$ ) gesteigert. Dieser Versuch wurde jeweils 3-mal für Muskel- und Fettgewebe durchgeführt. Die maximale Bestrahlungsdauer betrug $30 \mathrm{~s}$.

Um die Rolle der Sauerstoffkonzentration bei der Entstehung des Gewebebrandes zu ermitteln, und insbesondere um einen Schwellenwert zu finden, unter dem Entflammungen nicht vorkommen, wurde eine weitere Serie von jeweils 3 Versuchsdurchgängen angesetzt, bei denen das leichter entflammbare Fettgewebe unter Flutung der Versuchskammer mit $20 \mathrm{l} / \mathrm{min}$ Jetgas mit einer Laserleistung von $10 \mathrm{~W}$ bestrahlt wurde. Beginnend mit $21 \%$ wurde der Sauerstoffanteil im Jetgas in 5\%Schritten erhöht. Bestimmt wurden die Zeit bis zum ersten Aufflammen sowie die Anzahl Entflammungen/min unter einem Dauerstrahl im „Superpuls-Modus“.

\section{Ergebnisse}

$\mathrm{Zu}$ Beginn der Untersuchungsreihe wurde festgestellt, welche Entflammungs- und Brandphänomene zu erwarten waren, wenn ein genügend intensiver Laserstrahl mit einer Leistung von $10 \mathrm{~W}$ ohne Zeitlimite direkt auf das Gewebe gehalten wird. Die an verschiedenen Stellen wiederholten Applikationen ergaben folgende vier verschiedene Phänomene, die stets in dieser Reihenfolge auftraten:

1. Funkenbildung,

2. Rauchentwicklung,

3. kurzzeitiges Aufflammen und

4. anhaltender Brand.

Zunächst sollte festgestellt werden, inwieweit die oben beschriebenen Entflammungs- und Brandphänomene von der Laserleistung abhängig sind, und ob es dabei einen Unterschied zwischen Muskel- und Fettgewebe gibt. Im ersten Durchgang wurde Muskelgewebe für $30 \mathrm{~s}$ mit steigender Laserintensität von 2-15 W bestrahlt (• Abb. 1). Mit 2 und $4 \mathrm{~W}$ waren keinerlei Entflammungs- und Brandphänomene auslösbar. Funkenbildung trat ab 6 W, Rauchentwicklung ab $8 \mathrm{~W}$, vereinzeltes kurzes Aufflammen ab 


\section{Zusammenfassung $\cdot$ Abstract}

Anaesthesist 2006 ·5:541-546 DOI 10.1007/s00101-006-0974-y

c) Springer Medizin Verlag 2006

O. Juri · D. Frochaux · G. P. Rajan · P. Biro

Entflammungs- und Brandverhalten von biologischem Gewebe bei In-vitro-Bestrahlung mit dem $\mathrm{CO}_{2}$-Laser

\section{Zusammenfassung}

Fragestellung. Bei mikrolaryngoskopischen Eingriffen an den Atemwegen unter Anwendung von chirurgischen Laserstrahlen besteht eine Gefahr für Entflammungs- und Brandzwischenfälle. Selbst unter Weglassung von brennbaren Materialien liegt ein Restrisiko durch körpereigenes Gewebe vor, das unter der Einwirkung des Laserstrahls als partikelhaltiges Aerosol ("Lasersmog“) im Operationsgebiet entsteht. Diese Untersuchung bezweckt die möglichst realitätsnahe Simulation dieses Phänomens und die Feststellung der Rahmenbedingungen, in denen eine Entflammungsgefahr vorliegt.

Methodik. Zur Simulation des Operationsgebietes wurde in Anlehnung an die europäische Norm ISO-11990 eine zylinderförmige einseitig verschlossene Stahlkammer konstruiert, in die geeignete Schweinefleischstücke als Zielobjekt für die Laserbestrahlung eingeführt wurden. Mit einem Jetventilator wurde Beatmungsgas mit variabler Sauerstoffkonzentration in die Kammer eingelassen. Das Zielobjekt wurde mit Laserstrahlen unterschiedlicher Intensität und Zeitdauer im "Superpuls-Modus" (Impulsrate $250 \mathrm{~Hz}$ ) und mit einem Auftreffwinkel von $75^{\circ}$ bestrahlt. Die Laserleistung wurde von 2-15 W variiert, und Art, Dauer, Intensität sowie Häufigkeit von Entflammungs- und Brandphänomenen wurden dokumentiert.

Ergebnisse. Es traten Brandphänomene, beginnend mit Funkenbildung bis zu anhaltendem Brand, auf. Die Intensität dieser Brandphänomene war bei fetthaltigem Gewebe höher als bei Muskulatur. Diese nahm auch bei Steigerung der Laserleistung und der Bestrahlungsdauer zu. Als wichtigster Faktor wurde die Sauerstoffkonzentration identifiziert: Der niedrigste Wert, bei dem ein Aufflammen auftrat, war $35 \%$ nach $42 \mathrm{~s}$ bzw. $40 \%$ nach 20 s. Höhere Konzentrationen als
$60 \%$ führten fast sofort zu Funkenbildung und Aufflammen.

Schlussfolgerungen. Bei der Anwendung von laserchirurgischen Techniken für Atemwegseingriffe unter Jetventilation liegt selbst bei Verwendung laserresistenter Materialien ein Risiko von Brandkomplikationen vor. Der vom Laserstrahl freigesetzte Lasersmog aus dem operierten Gewebe kann in einer sauerstoffreichen Atmosphäre entflammt werden. Um diese Gefahr zu vermindern, sind eine möglichst tiefe Sauerstoffkonzentration im Beatmungsgas $(<40 \%)$, eine niedrigstmögliche Laserleistung $(<6 \mathrm{~W})$ und eine Begrenzung der Dauer der einzelnen Laserimpulse $(<10$ s) einzuhalten.

\section{Schlüsselwörter}

Atemwegseingriffe · Jetventilation · Brandgefahr $\cdot$ Lasersmog

\section{Ignition and burning of biological tissue under simulated $\mathrm{CO}_{2}$-laser surgery conditions}

\section{Abstract}

Background. Laser surgery in endoscopy has greatly enhanced the surgical armamentarium for treating various laryngeal and hypopharyngeal disorders, but harbours a risk of tissue ignition and inflammation of surgical and anaesthetic instrumentation. However, even if non-inflammable material is used, there is still a residual fire hazard from endogenous tissue that may develop an ignitable aerosol (so-called laser smog) as an effect of laser irradiation. The aim of this study was to investigate how tissue carbonisation and vaporisation contributes to the risk of airway fire.

Methods. For the simulation of gas accumulation in the hypopharynx and larynx following the European standard ISO-11990, a cylindrical steel chamber with an open and a closed end has been used to simulate the operative setting. Pork meat chunks with a tissue composition similar to the larynx and hy- popharynx such as fat, muscle, cartilage and bone were introduced into this chamber. Ventilation was achieved through jet ventilation with disposable, non-inflammable laser jet catheters. The tissue was then repeatedly exposed to a laser beam in super-pulse mode (pulse rate $250 \mathrm{~Hz}$ ) with various intensities and exposure lengths at an impact angle of $75^{\circ}$. The laser intensity was varied from 2 to $15 \mathrm{~W}$. The type, duration, intensity and incidence of tissue ignition were recorded and analysed.

Results. The degree of tissue ignition correlated with laser intensity. Low laser intensity caused spark formation whereas high intensity resulted in sustained tissue fire. The type of tissue had an impact on ignition intensity thereby showing lower ignition thresholds and higher ignition susceptibility in fat-containing tissue compared to muscle. The most important factor for occurrence of tissue igni- tion was the chamber oxygen concentration which displayed an inverse correlation with the time until tissue ignition. Oxygen concentrations of $35 \%$ led to tissue ignition in $42 \mathrm{~s}$, $40 \%$ oxygen in $20 \mathrm{~s}$. Oxygen concentrations higher than $60 \%$ resulted in immediate tissue ignition.

Conclusions. Despite the use of non-inflammable materials in endoscopic laser surgery of the upper airway and hypopharynx, the risk of tissue ignition remains due to the inflammable laser smog which is easily ignited in an oxygen-rich environment. Hence to minimise this risk, we recommend using oxygen concentrations lower than $40 \%$, low laser intensities $(<6 \mathrm{~W})$ and limiting continuous laser activation to periods shorter than $10 \mathrm{~s}$.

Keywords

Airway surgery - Jet ventilation - Fire hazzard . Laser smog 

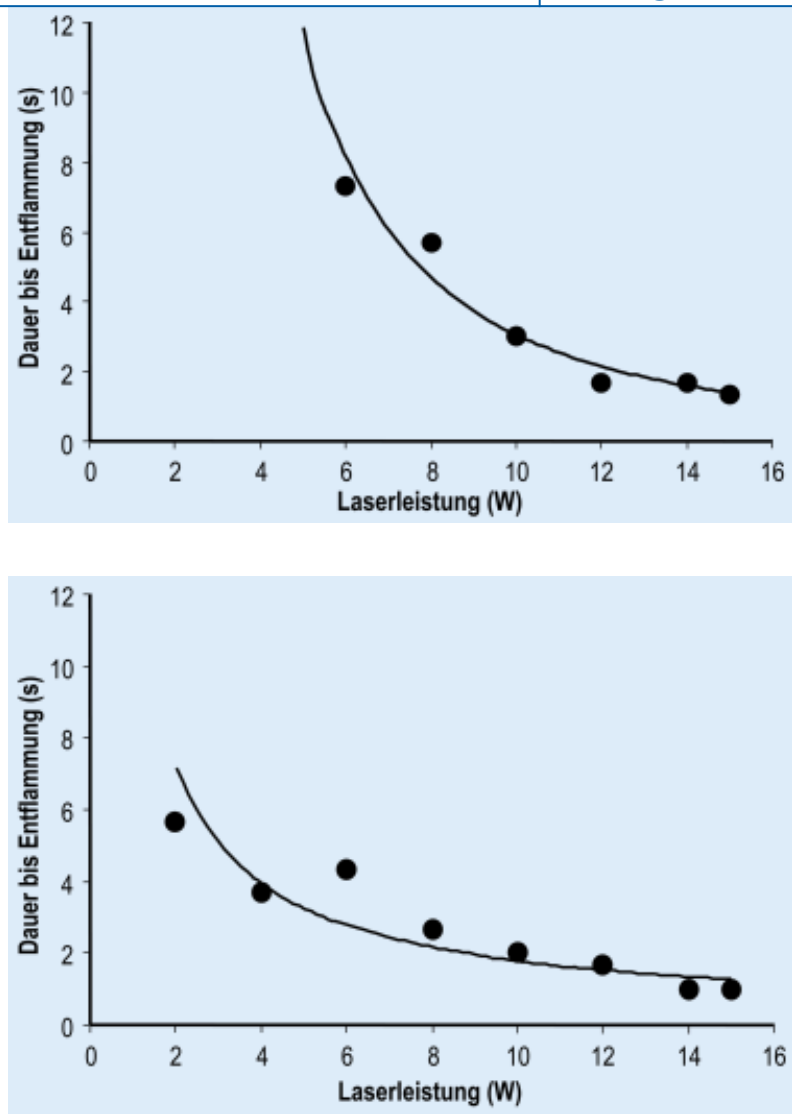

Abb. $3 \backsim$ Dauer bis zum Auftreten einer Entflammung (Mittelwerte) bei Muskelgewebe in Abhängigkeit von der Laserleistung. Rahmenbedingungen: $75^{\circ}$-Auftreffwinkel auf Gewebe, Gasfluss durch den Jetkatheter $20 \mathrm{l} /$ min, Sauerstoffkonzentration 100\% (Trendlinie vom Typ „potenziell“ durch Excel eingefügt)
Abb. $4<$ Dauer bis zum Auftreten einer Entflam- mung (Mittelwerte) bei Fettgewebe in Abhängig- keit von der Laserleistung. Rahmenbedingungen: $75^{\circ}$ - Auftreffwinkel auf Gewe- be, Gasfluss durch den Jet- katheter $20 \mathrm{l} / \mathrm{min}$, Sauer- stoffkonzentration $100 \%$. (Trendlinie vom Typ „po- tenziell" durch Excel ein- gefügt)

$10 \mathrm{~W}$ auf. Ab $12 \mathrm{~W}$ konnte man anhaltende Brände in Form von mehrere Zentimeter langen Stichflammen auslösen, die ohne weitere Laserbestrahlung so lange brannten, wie die Sauerstoffzufuhr andauerte. Die gleiche Versuchskonfiguration im benachbarten Fettgewebe führte erwartungsgemäß bei geringerer Laserintensität zu vehementeren Entflammungs- und Brandphänomenen. Bereits bei $2 \mathrm{~W}$ war Funkenbildung aufgetreten; kurzes Aufflammen war bereits bei $8 \mathrm{~W}$ zu beobachten. Die selbst unterhaltenden Brände unter anhaltender Sauerstoffzufuhr kamen jedoch ebenfalls nur mit Laserleistungen ab $12 \mathrm{~W}$ zustande (- Abb. 2).

Bei der Bestrahlung von Muskelgewebe betrug die niedrigste Laserleistung $6 \mathrm{~W}$, die ein Aufflammen nach durchschnittlich $7 \mathrm{~s}$ auslösen konnte (• Abb. 3). Höhere Energien führten zu früherem Aufflammen, bis zu $1 \mathrm{~s}$ bei $15 \mathrm{~W}$. Beim analogen Vorgehen mit dem leichter entflammbaren Fettgewebe, konnten wir bereits nach durchschnittlich $5 \mathrm{~s}$ mit $2 \mathrm{~W}$ ein kurzes Aufflammen provozieren (• Abb. 4).

Um die Rolle der Sauerstoffkonzentration zu ermitteln, wurde Fettgewebe mit einer Laserleistung von $10 \mathrm{~W}$ und unter Flutung der Versuchskammer mit 20 1/ min Beatmungsgas bestrahlt. Als positives Entflammungsereignis wurde ein kurzes Aufflammen gewertet. In einer Versuchsserie wurde die Zeit bis zum ersten Aufflammen (• Abb. 5), in einer zweiten die Anzahl Entflammungen/ min bestimmt (• Abb. 6). Die niedrigste Sauerstoffkonzentration, bei der ein Aufflammen nach ca. $42 \mathrm{~s}$ aufgetreten ist, war $35 \%$. Diese Zeitdauer verkürzte sich auf 20 s bei $40 \%$. Höhere Konzentrationen als $60 \%$ führten fast sofort zu einem Aufflammen. Bei der Bestimmung der Anzahl Entflammungen/Zeiteinheit ergaben sich 5 Entflammungen/min bei einer Sauerstoffkonzentration von 40-50\%, während bei höheren Sauerstoffkonzentrationen die Häufigkeit rapide bis zu einem Maximum von ca. 50 Entflammungen/ min anstieg.

\section{Diskussion}

Üblicherweise sind es die von außen zugeführten Materialien, die bei Laseranwendung mit einem Entflammungs- oder Brandrisiko verbunden sind. Die Beschäf- tigung mit diesem Problem hat dazu geführt, dass zunehmend bessere und sicherere Materialien entwickelt wurden [6], aber auch dazu, dass die endogenen Brandursachen weniger Aufmerksamkeit auf sich gezogen haben. Erstaunlicherweise ist Werkhaven in seiner Arbeit von 2004 der Ansicht, dass von körpereigenem Gewebe keine Entflammungsgefahr ausgeht, bzw. dass dieses Risiko rein theoretischer Natur sei [18]. Andere Autoren dagegen meinen, dass die unter der Bezeichnung "Lasersmog“ subsumierten Aerosole, die von dehydriertem und karbonisiertem Eigengewebe des Patienten stammen, unter bestimmten brandfördernden Bedingungen ein erhebliches Risiko für Brandzwischenfälle darstellen $[9,17]$. Diese Untersuchung dient dem Nachweis des Phänomens der Lasersmogentflammung und versucht die Rolle der beteiligten Einflussfaktoren zu quantifizieren.

Die in vitro simulierten Bedingungen des Versuchsaufbaus sind durchaus vergleichbar mit denjenigen, die im klinischen Alltag vorkommen. Es wurden lediglich die kritischen Versuchsbedingungen über das klinisch übliche Maß hinaus verändert, um die Grenzbedingungen herauszufinden, ab denen Entflammungs- und Brandphänomene mit hoher Wahrscheinlichkeit auftreten. Von entscheidender Bedeutung sind hierbei die Art des getroffenen Gewebes, die Intensität des verwendeten Laserstrahls, die Sauerstoffkonzentration in der unmittelbaren Umgebung und die Dauer der applizierten kontinuierlichen Bestrahlung $[9,16]$.

\section{Art des Gewebes}

Der Larynx und die angrenzenden Strukturen bestehen überwiegend aus Knorpelgewebe. Unter der Schleimhaut findet sich außerdem Fett- und Muskelgewebe. Die zur Simulation verwendeten Fleischstücke haben eine vergleichbare Zusammensetzung aus Fett, Knorpel und Muskulatur, sodass man ein analoges Entflammungsund Brandverhalten annehmen darf. Muskel- und Knorpelgewebe erwiesen sich als weniger entflammbar als Fettgewebe. Bei Anwendung von explizit kritischen Rahmenbedingungen (steiler Auftreffwinkel von $75^{\circ}$, 30-s-Bestrahlungsdauer, Sauer- 
stoffzufuhr von $201 / \mathrm{min}$ ) konnten Entflammungen von Muskelgewebe erst mit einer Laserleistung von $10 \mathrm{~W}$ und mehr ausgelöst werden (• Abb. 1). Fettgewebe entzündete sich unter den gleichen Rahmenbedingungen bereits bei $8 \mathrm{~W}$, und selbst mit $2 \mathrm{~W}$ war bereits Funkenbildung zu beobachten (• Abb. 2).

\section{Intensität des Laserstrahls}

Mit zunehmender Intensität des Laserstrahls nimmt die Häufigkeit von Brandphänomenen $\mathrm{zu}$, während die Zeitdauer bis zum Auslösen dieser Effekte kürzer wird. Bei Muskelgewebe ist unter den oben erwähnten „kritischen Rahmenbedingungen“ lediglich eine Laserintensität von $<5 \mathrm{~W}$ als relativ entflammungssicher anzusehen (- Abb. 3). Fettgewebe dagegen kann selbst mit der sehr niedrigen Intensität von $2 \mathrm{~W}$ entflammt werden (• Abb. 4).

\section{Sauerstoffkonzentration}

Die für diesen Parameter angewendeten Rahmenbedingungen wurden, wie folgt, festgelegt: Fettgewebe, steiler Auftreffwinkel von $75^{\circ}$, Gasfluss $201 / \mathrm{min}$, Laserleistung $10 \mathrm{~W}$ und Bestrahlungsdauer $45 \mathrm{~s}$. Hierbei liegt die kritische Sauerstoffkonzentration, bei der es zu Entflammungen kommen kann bei $\geq 30 \%$ (• Abb. 5 und 6). Durch Begrenzung der Impulsdauer auf $<30$ s kann diese Grenze als $\geq 40 \%$ angesehen werden. Das heißt aber nicht, dass bei niedrigeren Sauerstoffkonzentrationen grundsätzlich keine Entflammungen möglich wären; lediglich ihre Wahrscheinlichkeit ist reduziert [10]. Das bei der Jetventilation grundsätzlich bestehende Phänomen des „entrainment", $d$. h. die Beimischung von angesaugter Umgebungsluft zum Beatmungsgas, verursacht eine Verminderung der Sauerstoffkonzentration im Atemweg, wenn ein Wert über $21 \%$ im Frischgas eingestellt war. Die Ausprägung dieses Effektes ist individuell schwer vorhersehbar, da dies von verschiedenen z. T. inkonstanten Faktoren abhängt [1]. Vom Standpunkt der Brandgefahr handelt es sich hierbei jedenfalls um einen günstigen, wenngleich wenig berechenbaren Effekt.

Abb. 5 Dauer bis zum Auftreten einer Entflammung (Mittelwerte) bei Fettgewebe in Abhängigkeit von der Sauerstoffkonzentration. Rahmenbedingungen: $75^{\circ}$-Auftreffwinkel auf Gewebe, Gasfluss durch den Jetkatheter $20 \mathrm{l} / \mathrm{min}$, Laserleistung $10 \mathrm{~W}$ (Trendlinie vom Typ ",potenziell“ durch Excel eingefügt)

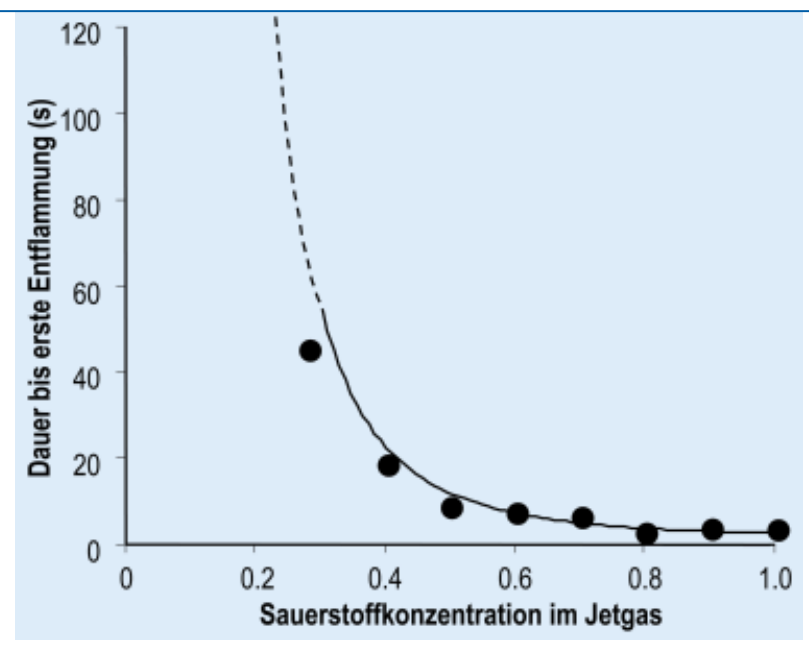

Abb. 6 Anzahl von Entflammungen/Bestrahlungsminute (Mittelwerte) bei Fettgewebe in Abhängigkeit von der Sauerstoffkonzentration. Rahmenbedingungen: $75^{\circ}$-Auftreffwinkel auf Gewebe, Bestrahlungsdauer $30 \mathrm{~s}$, Gasfluss durch den Jetkatheter $20 \mathrm{l} / \mathrm{min}$, Laserleistung $10 \mathrm{~W}$ (Trendlinie vom Typ „potenziell“ durch Excel eingefügt)

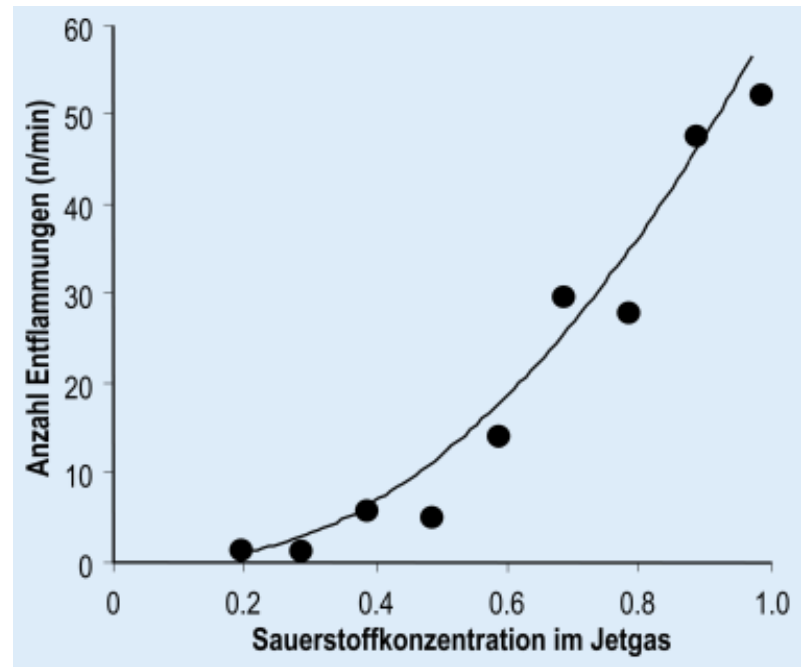

\section{Dauer der applizierten} kontinuierlichen Bestrahlung

Wenn man in Betracht zieht, dass in der klinischen Anwendung die oben beschriebenen „kritischen Rahmenbedingungen“ in der Regel nicht erreicht werden, erst recht nicht alle zur gleichen Zeit, dann kann man durchaus einigermaßen sichere Grenzbedingungen definieren. Freilich ist auch dann vom Brandverhalten des am meisten zur Entflammung neigenden Fettgewebes auszugehen, da dieses praktisch in allen Operationsfeldern vorkommt. $\mathrm{Zu}$ empfehlen ist, die Impulsdauer auf maximal $10 \mathrm{~s}$ zu begrenzen [4]; hierbei reichen bereits sehr kurze Pausen von jeweils 3 s aus, um das bestrahlte Gewebe abkühlen zu lassen und einen neuen Impuls auslösen $\mathrm{zu}$ dürfen.

\section{Zusammenschau}

Es sollte mit der niedrigstmöglichen Laserleistung gearbeitet werden, die eine saubere Schnittführung und eine ausreichende Koagulation ermöglicht. Dies ist durchaus mit Einstellungen von $6 \mathrm{~W}$ und weniger der Fall, wie sie auch meistens klinisch verwendet werden [13]. Eine Begrenzung der Dauer der einzelnen Laserimpulse (<10 s) empfiehlt sich. Schlussendlich sollte die Sauerstoffkonzentration im Frischgas nicht über 40\% liegen, solange der Laser aktiviert ist $[1,14,17,18]$. Wenn Letztere aus Gründen einer ungenügenden Oxygenierung nicht ausreichen sollte, können während der Operation bestrahlungsfreie Intervalle mit hoher Sauerstoffkonzentration eingeschaltet werden, bis die pulsoxymetrische Sauerstoffsättigung auf ein akzeptables Niveau angehoben worden ist. In jedem Fall ist beim 
Sauerstoffanteil grundsätzlich das Prinzip so hoch wie nötig und so tief wie möglich anzuwenden.

Die Wahrscheinlichkeit eines Atemwegsbrandes aufgrund von körpereigenem Gewebe allein ist kleiner als von eingebrachtem brennbaren Material und aufgrund der geringeren Menge des Brennstoffs auch von geringerer Ausprägung und Gefährlichkeit. Falls es trotzdem zu einer Entflammung von Lasersmog kommt, sind gleichermaßen Sofortmaßnahmen zur Unterbrechung des Brandes, der Beseitigung der Ursache und der Minimierung der Brandfolgen einzuleiten, wie sie andernorts zutreffend und ausführlich beschrieben wurden $[8,18]$.

\section{Fazit für die Praxis}

Bei der mikrolanryngoskopischen Laserchirurgie der Atemwege unter Jetventilation besteht selbst bei Verwendung von sog. laserresistenten Materialien ein Restsrisiko von Entflammungsund Brandkomplikationen. Dieses rührt von freigesetzten Partikeln aus dem operierten Gewebe des Patienten (Lasersmog), die in einer sauerstoffreichen Atmosphäre entflammt werden können. Um diese Brandgefahr gering zu halten, sind eine möglichst niedrige Sauerstoffkonzentration im Beatmungsgas ( $<40 \%)$, eine ebenfalls niedrigstmögliche Laserleistung ( $<6$ W) und eine Begrenzung der Dauer der einzelnen Laserimpulse $(<10$ s) einzuhalten.

\section{Korrespondierender Autor \\ PD Dr. P. Biro}

Institut für Anästhesiologie, Universitätsspital Rämistraße 100, 8091 Zürich

peter.biro@usz.ch

Interessenkonflikt. Der korrespondierende Autor weist auf eine Verbindung mit folgender Firma/Firmen hin: P. Biro war an der Entwicklung des LaserJet ${ }^{\circledR}$ Katheters beteiligt (s. [6]). Es bestehen keine finanziellen Interessen.

\section{Literatur}

1. Arnold JE, Allphin AL (1992) Effect of extraluminal oxygen on carbon dioxide laser ignition of endotracheal tubes. Arch Otolaryngol Head Neck Surg 118: 722-724

2. Biro P, Wiedemann K (1999) Jetventilation und Anästhesie für diagnostische und therapeutische Eingriffe an den Atemwegen. Anaesthesist 48: 669685

3. Burgess GE, Le Jeune FE (1979) Endotracheal tube ignition during laser surgery of the larynx. Arch Otolaryngol 195: 561-562

4. Davis RK, Simpson GT (1983) Safety with the carbon dioxide laser. Otolaryngol Clin North Am 16: 801-813

5. Eckel HE (1997) Endoscopic laser resection of supraglottic carcinoma. Otolaryngol Head Neck Surg 117: 681-687

6. Frochaux D, Rajan GP, Biro P (2004) Verhalten des neuen LaserJet ${ }^{\circ}$-Katheters bei $\mathrm{CO}_{2}$-Laser-Anwendung unter simulierten klinischen Bedingungen. Anaesthesist 53: 820-825

7. Hermens JM, Bennett MJ, Hirshman CA (1983) Anesthesia for laser surgery. Anesth Analg 62: 218-229

8. Lierz P, Heinatz A, Gustorff B, Felleiter P (2002) Management of intratracheal fire during laser surgery. Anesth Analg 95: 502

9. Padosch A, Polarz H (2001) Anästhesiologisches Management bei laserchirurgischen Eingriffen in der Hals-Nasen-Ohren-Heilkunde. Anaesthesist 50: 721-737

10. Paes ML (1987) General anaesthesia for carbon dioxide laser surgery within the airway. $\mathrm{Br} J$ Anaesth 59: $1610-1620$

11. Rudert HH, Werner JA (1995) Endoscopic resections of glottic and supraglottic carcinomas with the $\mathrm{CO}_{2}$ laser. Eur Arch Otorhinolaryngol 252: 146148

12. Santos P, Ayuso A, Luis M, Martinez G, Sala X (2000) Airway ignition during $\mathrm{CO}_{2}$ laser laryngeal surgery and high frequency jet ventilation. Eur J Anaesthesiol 17: 204-207

13. Sataloff RT, Spiegel JR, Hawkshaw M, Jones A (1992) Laser surgery of the larynx: the case for caution. Ear Nose Throat J 71: 593-595

14. Sesterhenn AM, Dunne AA, Braulke D, Lippert BM, Folz BJ, Werner JA (2003) Value of endotracheal tube safety in laryngeal laser surgery. Lasers Surg Med 32: 384-390

15. Vilaseca-Gonzalez I, Bernal-Sprekelsen M, BlanchAlejandro JL, Moragas-Lluis M (2003) Complications in transoral $\mathrm{CO}_{2}$ laser surgery for carcinoma of the larynx and hypopharynx. Head Neck 25: 382-388

16. Wainwright AC, Moody RA, Carruth JA (1981) Anaesthetic safety with the carbon dioxide laser. Anaesthesia 36: 411-414

17. Wegrzynowicz ES, Jensen NF, Pearson KS, Wachtel RE, Scamman FL (1992) Airway fire during jet ventilation for laser excision of vocal cord papillomata. Anesthesiology 76: 468-469

18. Werkhaven JA (2004) Microlaryngoscopy-airway management with anaesthetic techniques for $\mathrm{CO}_{2}$ laser. Pediatr Anesth 14: 90-94

\section{Die Deutsche Stiftung Organtransplantation (DSO) stellt ihren erweiterten Internet-Auftritt vor}

Die Homepage der DSO unter www.dso.de ist seit über fünf Jahren ein wichtiges Internetportal für die gezielte Suche nach Informationen zur Organspende und Transplantation. Jetzt stehen neue Menüpunkte, Themen und Serviceangebote zur Verfügung:

Ein Index erleichtert die Suche nach bestimmten Themenbereichen. Ein Newsletter informiert Interessierte über Aktualisierungen und die barrierefreie Seite ermöglicht mehr Nutzern den Zugang zu Informationen zum Thema Organspende und Transplantation. Zudem stehen monatlich aktualisierte Daten zur Zahl der Organspender in Deutschland zur Verfügung.

Der neue Menüpunkt „Fachinformationen" bietet - insbesondere für das ärztliche und pflegerische Personal auf den Intensivstationen in Krankenhäusern - detaillierte Informationen zu den relevanten Fragestellungen bei der Einleitung und Durchführung einer Organspende.

Darüber hinaus gibt es neue Seiten für Angehörige von Verstorbenen, die ihre Organe nach dem Tod gespendet haben. Hier erhalten Interessierte ausführliche Antworten auf häufige Fragen sowie Hinweise auf Selbsthilfegruppen, informative Internetseiten und Buchtipps zum Thema Trauer, Organspende und Transplantation.

Quelle: Deutsche Stiftung Organtransplantation (DSO), www.dso.de 